\title{
BMJ Open Quality A local quality initiative to improve follow-up times for patients with heart failure
}

\author{
Toni Schofield, ${ }^{1}$ Juan Duero Posada, ${ }^{1}$ Farid Foroutan, ${ }^{1}$ Ana Carolina Alba, ${ }^{1}$ \\ Michael McDonald, ${ }^{1}$ Meredith Linghorne ${ }^{2}$
}

To cite: Schofield T, Duero Posada J, Foroutan F, et al. A local quality initiative to improve follow-up times for patients with heart failure.BMJ Open Quality 2017;6:e000052. doi:10.1136/ bmjoq-2017-000052

Received 13 March 2017 Revised 22 June 2017 Accepted 2 August 2017

\section{CrossMark}

${ }^{1}$ Department of Medicine, Division of Cardiology, University of Toronto, Toronto, Ontario, Canada

${ }^{2}$ Ted Rogers Centre for Heart Research, Toronto, Ontario, Canada

Correspondence to Dr Toni Schofield, Department of Cardiology, Toronto General Hospital, Toronto, Ontario, M5G 2C4, Canada;

toni.schofield@uhn.ca

\section{ABSTRACT}

Introduction Heart failure is the most common cause of hospital admission in patients $>65$ years and around $50 \%$ of patients will be readmitted within 6 months. Inability to achieve timely outpatient follow-up may contribute to the high rates of avoidable rehospitalisation for this group of patients. Canadian guidelines recommend patients with heart failure should be seen within 14 days of discharge. Methods An audit demonstrated that less than half of advanced heart failure patients were being followed up within 14 days. In an effort to improve postdischarge follow-up in our heart function clinic, we used process mapping and applied a series of iterative changes to the appointment booking system using Plan-Do-Study-Act cycles to reduce waste and standardise.

Results The primary outcome measure, tracked over a period of 20 months, was percentage of patients booked within 14 days. At baseline, $37 \%$ of patients were seen within 14 days. After our series of interventions related to streamlining and standardising the appointment booking process, $77 \%$ of patients were seen within 14 days and $100 \%$ of patients were seen within 21 days.

Conclusion The changes made to the appointment booking process were reproducible, sustainable, effective and required no additional resources or funding.

\section{INTRODUCTION}

\section{Local problem and rationale}

At our institution, patients with acute decompensated heart failure ( $\mathrm{HF}$ ) can be admitted to General Cardiology or Internal Medicine. Of those admitted to Cardiology, a smaller number are managed directly by the $\mathrm{HF}$ service. These patients are generally younger, with more advanced disease and being evaluated for advanced therapies such as left ventricular assist devices or transplantation.

We noticed that we were not always meeting the Canadian Cardiovascular Society (CCS) guidelines for follow-up within 14 days. Patients were either being seen an extended time after discharge, or being readmitted before their next clinic appointment, and alarmingly, occasional patients reported not receiving an appointment at all and following up themselves with the clinic. The method for booking appointments was non-standardised and unclear. An appointment request was sent to a centralised fax number or to an email address that was accessed by several staff. There was no communication back to the requesting provider that the fax/email had been received or processed and patients were leaving hospital trusting that someone would call them or send them an appointment in the mail.

\section{Available knowledge}

$\mathrm{HF}$ is a chronic disease of epidemic proportion. In Canada, there are an estimated 600000 people living with $\mathrm{HF}$ and 50000 new cases diagnosed each year. ${ }^{1}$ It is the most common reason for hospitalisation in people $>65$ years of age despite advances in $\mathrm{HF}$ pharmacotherapy and devices. Patients with HF have high rates of readmission quoted between $10 \%$ and $50 \%{ }^{2}$ and up to $75 \%$ of these may be avoidable. ${ }^{3}$ Readmissions are more prevalent in the period after hospital discharge as well as in in advanced disease, at the preterminal phase. ${ }^{4}$ Patients are vulnerable during transitions of care ${ }^{5}$ and problems can arise in the postdischarge period relating to the understanding of discharge instructions, medication changes and side effects, and the early identification of warning signs and symptoms. ${ }^{6}$ Emphasis has been placed on the timing of follow-up after recognition that nearly half of readmissions occur before the first ambulatory visit. ${ }^{7}$ Following patients in a timely manner in an ambulatory setting gives the care provider an opportunity to check for complications of treatment, titrate medications, reinforce activity limitations and lifestyle instructions and discuss goals of care. Moreover, timely access to care is one of the Institute of Medicine's 6 domains of quality targeted for healthcare improvement. ${ }^{8}$ Multidisciplinary heart function clinics provide this opportunity, are cost-effective and have been shown to reduce rehospitalisation and mortality. ${ }^{7} 9$ The use of multidisciplinary heart function clinics has been incorporated 


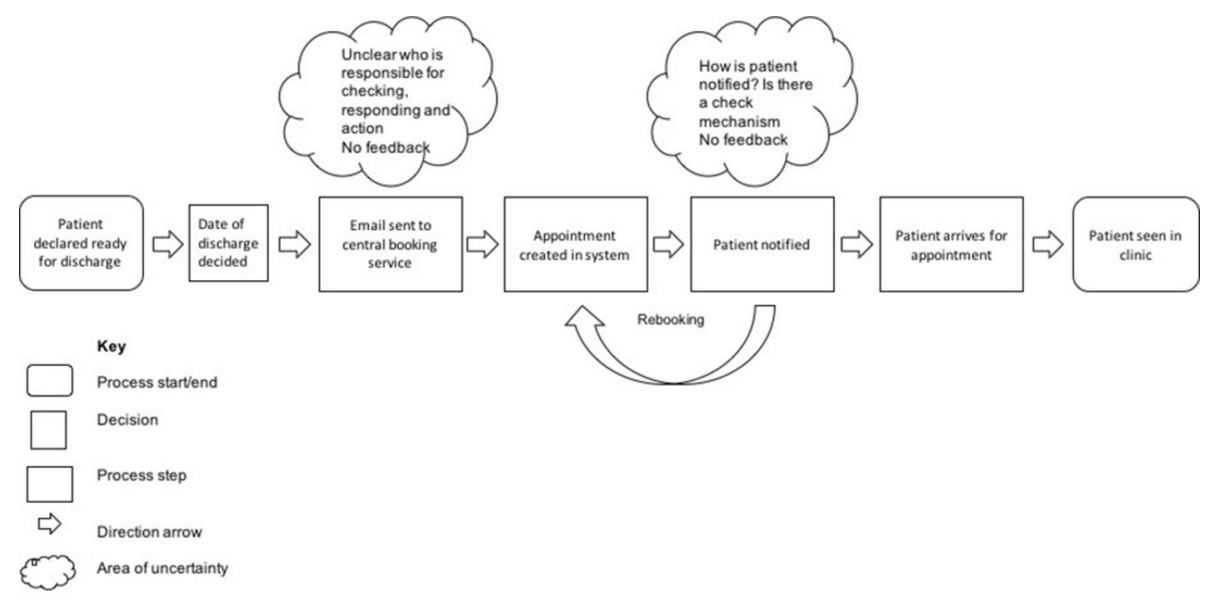

Figure 1 Process map of work flow before intervention.

into CCS guidelines along with the recommendation that patients be seen within 14 days $^{10}$.

\section{Aims}

The goal of this project was that over a 6-month period from September 2015 to March 2016, 90\% of eligible patients admitted to the inpatient HF team with decompensated HF will be seen in the multidisciplinary heart function clinic within 14 days of discharge.

\section{METHODS}

\section{Context}

Baseline data were extracted from retrospective audit of HF patients discharged between January and September 2015. The percentage of eligible patients seen within 14 and 21 days was recorded. Patients were considered ineligible for follow-up in our clinic if they were discharged to a long-term care facility, had a prognosis of $<3$ months or had local follow-up with a cardiologist outside of our clinic.

\section{Interventions and team}

The project was designed around the Model For Improvement, a quality improvement framework popularised by the Institute for Healthcare Improvement, that poses a series of three questions that address the scientific method: (1) what are we trying to accomplish? (2) how will we know a change is an improvement? (3) what changes can we make that will result in an improvement? These questions are addressed by applying three concepts: (a) the development of a timely and specific aim; (b) choosing a family of outcome, process and balancing measures; and (c) select interventions based on the underlying causes of the gaps in care. ${ }^{1112}$

A quality improvement team was formed comprising stakeholders from senior management (director of the heart failure service and unit manager), nurse practitioner champion (process owner), clinical fellows from the service, as well as quality improvement expert advice from co-learning curriculum seminars run by the Center for Quality Improvement and Patient Safety. Baseline data were communicated to senior staff in the cardiology department and support provided for this initiative.

A process map was constructed with key stakeholders from the ward and clinic to understand how patients are provided with follow-up on discharge (figure 1). This highlighted several areas of uncertainty where accountability could fail and the process becomes inefficient. The aim was to simplify, standardise the process and remove unnecessary duplication of work, while tracking the outcome measure. It was hypothesised that by standardising the workflow involving individuals who are usually part of the booking process we would see an increase in the percentage of patients given an appointment within 14 days.

\section{Study of the interventions}

The intervention was planned to use existing staff and technology resources, with no additional funding or infrastructure required. The interventions were conducted over a 6-month period from September 2015 to March 2016 with ongoing data collection after the interventions to assess sustainability. Monthly data of number of discharges from the service, proportion of eligible patients and those followed up in the clinic within 14 and 21 days were recorded in a database, and plotted on a run chart. Fishers Exact probability test was used to compare the proportions of patients followed up within 14 days preintervention and postintervention.

\section{Measures}

The primary outcome measure was the percentage of patients followed in the HF clinic within 14 days. The percentage of patients seen within 21 days was also tracked. Other balancing measures included clinic saturation, appointmentavailability and additional perceived workload.

\section{Analysis}

Data were plotted on a run chart using a statistical software package and analysed according to run chart rules published in 'The Healthcare Data Guide'. ${ }^{12}$ The 


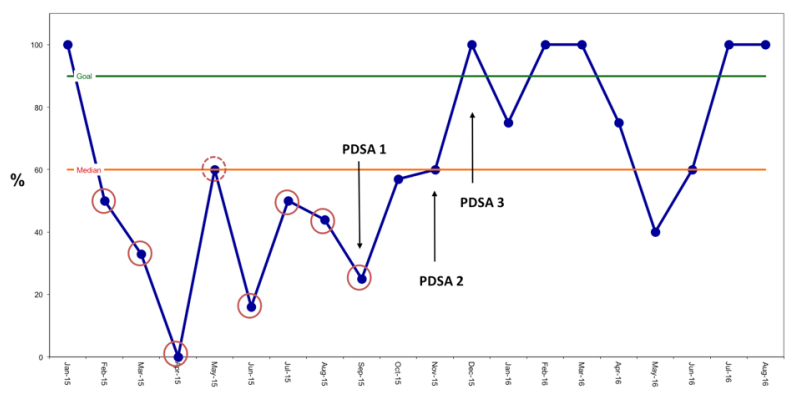

Figure 2 Run chart of the percentage of patients seen within 14 days.

manuscript was prepared in accordance with the SQUIRE V.2.0 guidelines. ${ }^{13}$

\section{Ethical considerations}

Research ethics board approval was not sought on the basis that this was a localised quality improvement project with no potential harm to patients.

\section{Study design}

Process mapping and root cause analysis were conducted after baseline data collection, and discovered time delays in emails or faxes being sent or received, lack of clear accountability among six administrative assistants for the checking and booking of requests created by a centralised email were the main problems. In addition, lack of space in regularly scheduled clinics was also an issue.

The booking process was restructured in a series of Plan-Do-Study-Act (PDSA) cycles:

PDSA 1. Initially, emails were sent to all attending staff, nurse practitioners and fellows who care for patients in the heart function clinic for educating and reminding them of the CCS guidelines and of the aims of the project. The rationale for this was to engage healthcare providers in the project and remind them of the CCS follow-up Guidelines.

PDSA 2. The next change was to call the heart function clinic at the time of discharge to obtain an appointment for the patient. It was quickly discovered, that this strategy had several drawbacks: the clerks were not always able to answer the phone, necessitating a voice message and also resulted in increased interruptions and disruption to workflow. This led to the hypothesis for the next PDSA cycle; that if a clerk could be tasked to the booking of appointments for patients being discharged from the ward, it would lead to less disruptions overall and create accountability and standardisation around work processes.

PDSA 3. With the aim of reducing disruptions to workflow and reducing the amount of email and fax traffic to the clinic clerks, for this cycle, a dedicated clerk was tasked to attend the ward each day at a certain time, liaise with the unit clerk regarding pending discharges and book the patients into the clinic system. This was well received as it created less outstanding work for the clerks and resulted in immediate feedback for the nurse practitioner and patients regarding appointment time and date.

\section{RESULTS}

The effect of our interventions on the percentage of patients given an appointment within 14 and 21 days are demonstrated in figures 2 and 3 . As a result of the interventions, we observed an increase in the primary outcome measure of the percentage of patients given a follow-up appointment within 14 days postdischarge. Specifically, during the 9 months of baseline data collection between January and September 2015, 57 patients were discharged from our service. Of these, 53 (93\%) were eligible to be seen in our clinic, and only $20(38 \%)$ had an appointment within 14 days and 29 (55\%) within 21 days. After implementing the changes, the proportion seen within 14 days improved to $77 \%$ ( $\mathrm{p}$ value 0.01 ) and was mostly sustained over a 9-month period. All $(100 \%)$ patients after the interventions were seen within 21 days (figure 3); this change was sustained for a period of 9 months.

The run chart in figure 2 shows a goal line at $90 \%$ as per our aim statement and the primary outcome measure of the percentage of patients seen within 14 days from January 2015 to August 2016. The red line represents the median or centre line for the data. PDSA cycles are annotated. The run chart demonstrates that the rules for a shift are met with $>6$ consecutive points below the median in the data prior to November (red circles). Also, there are less runs crossing the centre line than would be expected for random variation alone $(<6$ runs implies significance in a dataset of 20 values: Health Care Data Guide 2011). ${ }^{12}$ Figure 3 demonstrates the changes in follow-up within 21 days, with centre line frozen prior to the interventions and extended after the interventions.

\section{DISCUSSION AND INTERPRETATION}

The aim of the project was to improve clinic follow-up times for patients with HF discharged from the advanced HF service. The results show that our interventions achieved a significant improvement in follow-up times. Despite our goal of $90 \%$ seen within 14 days not being met, a considerable improvement was achieved in proportion of patients seen within 14 days and all eligible patients seen within 21 days.

One of the advantages of conducting a localised project was that we were able to engage all members of the team and had complete control over the processes we were trying to change.

The time spent understanding the current processes was valuable, as it allowed solutions to be targeted to areas of opportunity to remove waste (waiting, overprocessing and motion of information), uncertainty and to standardise. Though satisfaction was not quantitatively assessed, it was generally agreed that booking and 


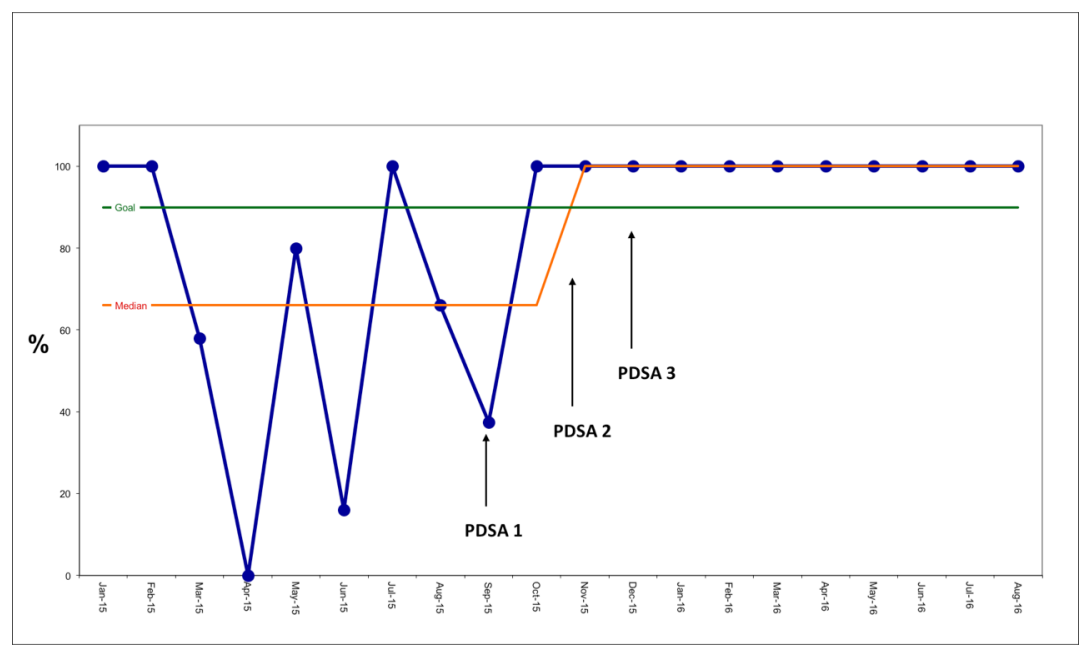

Figure 3 Run chart of the percentage of patients seen within 21 days.

communicating appointments directly was of benefit to the patient and caregiver, process owner and clinic staff.

\section{LESSONS AND LIMITATIONS}

PDSA 1, an educational intervention, generally considered to be 'low impact' on the hierarchy of effectiveness, (Institute for Safe Medication Practices 1999) was an essential step in enhancing stakeholder engagement and raise awareness of the rationale for the project. The nature of PDSA methodology and rapid cycle changes allowed for swift recognition that PDSA 2 was having a negative impact on administrative staff due to interruptions to workflow caused by the calls to book appointments and so it could be addressed with the next change. PDSA 3 involved using an administrative clerk as the booking modality. The team was cognizant that reliance on a single individual had risks to sustainability and strategies were considered to mitigate against this risk. The work was presented at a departmental symposium and the team proposed that unit clerks be trained in using the booking system so that they may then do this directly as part of their work flow on discharging a patient. This would prevent the booking clerk from having to physically visit the ward to do this, and would also address the problem of reliance on one individual. This change would require more senior engagement, interdepartmental involvement and additional resources.

Limitations of our project included relatively small numbers of patients discharged monthly from the service. In figure 2, there was a drop in 14-day follow-up in January due to patient factors affecting the follow-up of one patient and another observed decrease in percentage follow-up in April, May and June, which subsequently recovered in July and August. The team had not implemented any new changes in this period and propose that clinic space was limited due to a combination of staff leave and international conferences during this period, causing clinic back log. All patients however were still seen within 21 days (figure 3 ).
Although the goal of achieving $90 \%$ follow-up within 14 days was not realised in the project time frame, we were able to effect significant improvement on the percentage of patients followed up at 14 days and achieve $100 \%$ follow-up within 21 days. This demonstrates the value of a departmental change initiative on the attitudes of all members of the department and run-off effects that even 'low effectiveness' interventions such as education, communication and awareness can have.

Ongoing change ideas, after training the unit clerks, would be to extend out to the general cardiology ward. Of course, there could be barriers to trying to mandate a 14-day follow-up policy, such as saturation of clinics, or limited access to certain clinicians. Another idea we are exploring is implementing follow-up phone calls to risk stratify patients in the early post discharge period to determine who would benefit from earlier follow-up.

On reflection, additional measures could have been implemented during the project to formally assess patient-centred experience and satisfaction around care transitions in addition to tracking other institutional benchmarks of quality such as readmission rates and impact on length of stay.

\section{CONCLUSION}

There is evidence in the literature to suggest that achieving timely follow-up for patients with HF can reduce avoidable hospital readmissions and improve care. We identified an opportunity for improvement in 14-day follow-up in our advanced HF service. Process mapping and root cause analysis identified areas to target improvement efforts. Using the model for improvement and a series of PDSA cycles we were able to achieve an increase in the percentage of patients being seen within 14 days from $38 \%$ preintervention to $77 \%$ after implementation of the changes and $100 \%$ of patients seen within 21 days. A run chart was constructed for the main outcome measure and did imply a relationship between our interventions and change in outcome measure. This was further validated 
using Fishers Exact test on our pooled preintervention and postintervention follow-up proportions. We did this with no additional staffing or resources. Future endeavours are focused on scaling our change ideas to the rest of the cardiology service and telephone triage of follow-up services to make it more sustainable over the long term.

The authors are happy to be able to report that at the time of writing, some unit clerks are now trained to book appointments and a new electronic discharge template is able to 'pull' appointments directly from the booking system, so the patient has an appointment date and a contact number for the clinic in writing on the discharge summary.

The team gained experience in practical applications of quality improvement methodology and have a shared sense of achievement and departmental cohesion from the successful changes brought about by the project. There were no hard savings to demonstrate during the course of the project, however senior leadership can see the potential for savings over the long term with the formation of a robust follow-up system (eg, in reducing length of stay or reducing readmissions).

In the spirit of continuous improvement, some team members have gone on to enhance their knowledge as it relates to quality improvement and patient safety with recognised training programme and higher degrees and there are several other departmental projects underway to improve processes and outcomes for patients and their families living with HF.

Acknowledgements The authors would like to acknowledge and thank the patients and caregivers of the Peter Munk Cardiac Center, Toronto General Hospital and the help of Professor Heather Ross, Dr Brian Wong, Dr Edward Etchells, Tosha Samuel and Emolyne Marquez.

Contributors ML, TS and JDP: conducted the study participated in data collection, analyzed data and made iterative changes. TS, ACA and FF: analyzed the data overall and contributed to extra data collection. All authors: contributed to the editing of the paper. TS: submitted the study.

Competing interests None declared.
Provenance and peer review Not commissioned; externally peer reviewed.

Open Access This is an Open Access article distributed in accordance with the Creative Commons Attribution Non Commercial (CC BY-NC 4.0) license, which permits others to distribute, remix, adapt, build upon this work non-commercially, and license their derivative works on different terms, provided the original work is properly cited and the use is non-commercial. See: http://creativecommons.org/ licenses/by-nc/4.0/

(c) Published by the BMJ Publishing Group Limited. For permission to use (where not already granted under a licence) please go to http://www.bmj.com/company/ products-services/rights-and-licensing/

\section{REFERENCES}

1. Heart and Stroke Foundation. Heart failure. 2017 http://www. heartandstroke.ca/heart/conditions/heart-failure (accessed 10 Feb 2017).

2. Joynt KE, Jha AK. Who has higher readmission rates for heart failure, and why? Implications for efforts to improve care using financial incentives. Circ Cardiovasc Qual Outcomes 2011;4:53-9.

3. van Walraven $C$, Bennett $C$, Jennings $A$, et al. Proportion of hospital readmissions deemed avoidable: a systematic review. CMAJ 2011;183:E391-402.

4. Desai AS, Stevenson LW. Rehospitalization for heart failure: predict or prevent? Circulation 2012;126:501-6.

5. Krumholz HM. Post-hospital syndrome--an acquired, transient condition of generalized risk. N Engl J Med 2013;368:100-2.

6. Albrecht JS, Gruber-Baldini AL, Hirshon JM, et al. Hospital discharge instructions: comprehension and compliance among older adults. J Gen Intern Med 2014;29:1491-8.

7. Hernandez AF, Greiner MA, Fonarow GC, et al. Relationship between early physician follow-up and 30-day readmission among Medicare beneficiaries hospitalized for heart failure. JAMA 2010;303:1716-22.

8. Institute of Medicine. Crossing the quality chasm: a new health system for the 21st century. Washington, DC: The National Academies Press, 2001.

9. McAlister FA, Stewart S, Ferrua S, et al. Multidisciplinary strategies for the management of heart failure patients at high risk for admission: a systematic review of randomized trials. J Am Coll Cardiol 2004;44:810-9.

10. Howlett JG, Chan M, Ezekowitz JA, et al. The canadian cardiovascular society heart failure companion: bridging guidelines to your practice. Can J Cardiol 2016;32:296-310.

11. The Institute for Healthcare Improvement. How to improve. http:// www.ihi.org/resources/Pages/Howtoimprove/default.aspx

12. Lloyd P, Provost SKM. The heath care data guide: learning from data for improvement. 1st edn. San Francisco: Jossey-Bass, 2011.

13. Ogrinc G, Davies L, Goodman D, et al. SQUIRE 2.0 (Standards for QUality Improvement Reporting Excellence): revised publication guidelines from a detailed consensus process. Jt Comm J Qual Patient Saf 2015;41:474-9. 